Methodology All women with non-endometrioid EC, defined as serous, clearcell, carcinosarcoma and undifferentiated, were identified through the Swedish Quality Registry for Gynecological Cancer in the western Sweden health care region (1.9 million inhabitants) between 2010-2017 where the NGEC were implemented in 2013. Recurrences were identified including location and relative survival (RS), overall survival (OS) and disease-free survival (DFS) were analysed.The cohort was divided according to treatment protocol before and after NGEC implementation and compared.

Result(s)* In total 401 patients were identified and after exclusion for neoadjuvant chemotherapy, palliative treatment and preoperative stage IV, the final study cohort consisted of 261 patients who underwent primary surgical treatment with no evidence of disease at start of follow-up. The cohort before NGEC implementation was 103 patients and 158 patients after. The total recurrence rate was $26 \%$ and $6 \%$ were localized only to vagina. The RS rate for all patients diagnosed with a recurrence was $14.1 \%(95 \% \mathrm{CI} 7.7-26.0)$ compared to $92.8 \%(95 \% \mathrm{CI} 85.7-100.5)$ with no recurrence. Both the RS and OS rates were significantly improved after implementation of the NGEC. The 5-year RS was 58.8\% (95\%CI 48.6-71.0) for treatment in the first period and $79.8 \%$ (95\% CI 71.0 $89.8)$ for the second period $(p=0.005)$. The 5 -year OS was $54.3 \% \quad(95 \% \mathrm{CI} \quad 45.5-64.9)$ and $68.7 \% \quad(95 \% \mathrm{CI} \quad 61.3-77.0)$ respectively $(p=0.011)$.

Conclusion* In this populationbased study of a complete cohort of non-endometrioid ECs we conclude that adequate lymphnode staging followed by adjuvant chemotherapy to all patients and radiotherapy only to those with postive nodes is associated with superior survival compared to chemo-and radiotherapy to all regardless of lymphnode status.

\section{SENTINEL NODE, IN ENDOMETRIAL CANCER. OUR RESULTS}

F Fargas Fabregas*, R Fábregas. Barcelona, Barcelona, Spain

\subsection{6/ijgc-2021-ESGO.122}

Introduction/Background* the goal is the detection of the sentinel node in initial endometrial cancer, in our center; and compare the different techniques (blue, technetium and indocianin green)

Methodology All patients diagnosed with an early stage of endometrial cancer, who are performed sentinel node technique

The objective is to assess the detection capability of the sentinel node based on the tracer used, and the validity of this technique in low-risk tumors, in our center

Result(s)* we have recruited a total of 119 patients. Sentinel node detection results vary depending on the plotter used. the best results were those of the combination of blue with indocian green (91\%), as already described in the literature.

The number of positive nodes in this subgroup of patients (low risk) was very low.

Conclusion* Indocianin green is the best tracer, for sentinel node detection.

Although the number of positive nodes, in these patients, is very low, the low morbidity that presents the tecnique, we would not recommend the NO relaization of this technique, because we can perform a more specific analysis of these nodes.

\section{5 ENGOT-EN11/GOG-3053/KEYNOTE-B21: PHASE 3 STUDY OF PEMBROLIZUMAB OR PLACEBO + ADJUVANT CHEMOTHERAPY \pm RADIOTHERAPY FOR HIGH-RISK ENDOMETRIAL CANCER}

${ }^{1} \mathrm{~T}$ Van Gorp ${ }^{*},{ }^{2} \mathrm{MR}$ Mirza, ${ }^{3} \mathrm{~A}$ Lortholary, ${ }^{4} \mathrm{~B}$ Vergote, ${ }^{5} \mathrm{D}$ Cibula, ${ }^{6} \mathrm{~A}$ Walther, ${ }^{7} \mathrm{~A}$ Savarese, ${ }^{8} \mathrm{MP}$ Barretina-Ginesta, ${ }^{9} \mathrm{FU}$ Ortac, ${ }^{10} \mathrm{C}$ Papadimitriou, ${ }^{11} \mathrm{~L}$ Bodnar, ${ }^{12} \mathrm{CH}$ Lai, ${ }^{13} \mathrm{~K}$ Hasegawa, ${ }^{14} \mathrm{X}$ Xie, ${ }^{15} \mathrm{EL}$ Barber, ${ }^{16} \mathrm{R}$ Coleman, ${ }^{17} \mathrm{~J}$ Lichfield, ${ }^{18} \mathrm{~A}$ Grandhi, ${ }^{19} \mathrm{~B}$ Slomovitz. ${ }^{1}$ UZ Leuven, Leuven, Belgium; ${ }^{2}$ NSGO-CTU and Rigshospitalet, Copenhagen University Hospital, Copenhagen, Denmark; ${ }^{3}$ Centre Catherine de Sienne, Hôpital Privé du Confluent, Nantes, France; ${ }^{4} B G O G$ and University Hospitals Leuven, Leuven Cancer Institute, Leuven, Belgium; ${ }^{5}$ Department of Obstetrics and Gynecology, General Faculty Hospital in Prague, First Faculty of Medicine, Charles University, Prague, Czech Republic; ${ }^{6}$ Bristol Cancer Institute, University Hospitals Bristol, Bristol, UK; ${ }^{7}$ Istituto Nazionale Tumori Regina Elena, Rome, Italy; ${ }^{8}$ Catalan Institute of Oncology and Girona Biomedical Research Institute, Medical School University of Girona, Girona, Spain; ${ }^{9}$ Ankara University School of Medicine, Ankara, Turkey; ${ }^{10}$ Aretaieio University Hospital, National and Kapodistrian University of Athens, Athens, Greece; ${ }^{11}$ Department of Oncology and Immunooncology, Warmian-Masurian Cancer Center of the Ministry of the Interior and Administration's Hospital, Olsztyn, Poland; ${ }^{12}$ TGOG and Department of Gynecology and Obstetrics, Chang Gung Memorial Hospital, Linkou Branch, Taoyuan, Taiwan; ${ }^{13}$ Department of Gynecologic Oncology, Saitama Medical University, Hidaka, Saitama Prefecture, Japan; ${ }^{14}$ Women's Hospital, School of Medicine, Zhejiang University, Hangzhou, China; ${ }^{15}$ Northwestern University Feinberg School of Medicine, Chicago, IL, USA; ${ }^{16}$ US Oncology Research, The Woodlands, TX, USA; ${ }^{17}$ MSD, UK; ${ }^{18}$ Merck and Co., Inc., Kenilworth, NJ, USA; ${ }^{19}$ Broward Health, Fort Lauderdale, FL, USA

\subsection{6/ijgc-2021-ESG0.123}

Introduction/Background* Pembrolizumab, an anti-PD-1 antibody, has demonstrated activity in patients with previously treated mismatch repair (MMR) deficient (dMMR; 57.1\% objective response rate [ORR] as monotherapy and $63.6 \%$ ORR as combination therapy with lenvatinib) and MMR proficient (pMMR; 36.2\% ORR as combination therapy with lenvatinib) endometrial cancer. ENGOT-en11/GOG-3053/ KEYNOTE-B21 (NCT04634877) is a phase 3, randomized, double-blind study of pembrolizumab or placebo in combination with adjuvant chemotherapy with/without radiotherapy in patients with endometrial cancer.

Methodology Eligible patients are $\geq 18$ years old with newly diagnosed, high-risk (stage I/II non-endometrioid or with p53 abnormality and any histology, stage III/IVA), previously untreated endometrial cancer following surgery with curative intent with no evidence of disease post-operatively. Approximately 990 patients are randomized to receive pembrolizumab $200 \mathrm{mg}$ or placebo every 3 weeks (Q3W) for 6 cycles plus chemotherapy (carboplatin area under the curve [AUC] $5 / 6$ plus paclitaxel $175 \mathrm{mg} / \mathrm{m}^{2}$ Q3W or carboplatin AUC 2/ 2.7 plus paclitaxel $\left.60 \mathrm{mg} / \mathrm{m}^{2} \mathrm{QW}\right)$ in stage 1 . Patients receive pembrolizumab $400 \mathrm{mg}$ or placebo Q6W for 6 cycles in stage 2. Radiotherapy (external beam radiotherapy [EBRT] and/or brachytherapy) \pm radiosensitizing cisplatin $50 \mathrm{mg} / \mathrm{m}^{2}$ (days 1 and 29) may be administered after completion of chemotherapy. Randomization is stratified by MMR status (pMMR vs dMMR) and, within pMMR, by planned radiation therapy (cisplatin-EBRT vs EBRT vs no EBRT), histology (endometrioid vs non-endometrioid), and International Federation of Gynecology and Obstetrics surgical stage (I/II vs III/ IVA). Dual primary endpoints are disease-free survival (DFS; per investigator assessment) and overall survival (OS). Secondary endpoints include DFS (per blinded independent central review), DFS (per investigator assessment) and OS by biomarker status (PD-L1 and tumor mutational burden), safety (per National Cancer Institute Common Terminology Criteria for Adverse Events version 5.0), and quality of life (per European Organization for Research and Treatment of 
Cancer Quality of Life Questionnaire-Core 30 [EORTC QLQ-C30] and Endometrial Cancer Module [EORTC QLQEN24]). Enrolment began December 2020 and is ongoing at 221 sites in 28 countries.

Result(s)* N/A

Conclusion* N/A

\section{LAPAROSCOPIC REPAIR OF OBTURATOR NERVE INJURY AT THE TIME OF PELVIC LYMPHADENECTOMY}

R Linder*, J Gomez, T Feigenberg. trillium health partners, Gynecological Oncology , Canada

10.1136/ijgc-2021-ESG0.124

Introduction/Background* The obturator nerve is formed by the lumbar plexus, receiving its fibers from the anterior division of L2-4. Clinically, obturator nerve injury manifests with sensorial loss at the medial aspect of thigh, pain at medial portion of the groin and ipsilateral adductor weakness. The risk of obturator nerve injury is increased during pelvic lymphadenectomy and surgeries for gynecologic malignancies. Here, we present a video film of full-thickness transection of obturator nerve at the time of pelvic lymphadenectomy in a patient who underwent laparoscopic surgery for endometrial cancer. The transected nerve was primarily repaired through a laparoscopic approach at the time of surgery. The patient experienced a full recovery with no motor function deficiencies

Methodology

Result(s)* In this surgical film we present an 83-year-old woman, G2P2, who was diagnosed with a grade 3 endometrioid endometrial adenocarcinoma. She underwent a laparoscopic hysterectomy and sentinel lymph-node biopsies as well as resection of enlarged external iliac node. During the procedure, we identified a complete transection of the obturator nerve. To enable an end to end repair, the external iliac artery and vein were mobilized. The proximal end of the nerve was identified lateral to these vessels. An end-to-end anastomosis of the nerve was performed using Prolene sutures. The patient experienced an uneventful post-operative recovery and was discharge home on post-operative day 2, able to ambulate independently with no assistance required.

Conclusion* Obturator nerve injuries are a well-recognized complication of pelvic lymphadenectomy. Immediate laparoscopic nerve repair, can facilitate earlier motor recovery and prevent the need for laparotomy.

\section{UTILITY OF PELVIC MRI AND TUMOR MARKERS HE4 AND CA125 TO PREDICT DEPTH OF MYOMETRIAL INVASION AND CERVICAL INVOLVEMENT IN ENDOMETRIAL CANCER}

${ }^{1} \mathrm{~N}$ Zamani*, ${ }^{1} \mathrm{M}$ Modares, ${ }^{2} \mathrm{~F}$ Zamani. ${ }^{1}$ Tehran University of Medical Sciences, gynecologic oncology, Tehran, Iran; ${ }^{2}$ Tehran University of Medical Sciences, radiology, Tehran, Iran

\subsection{6/ijgc-2021-ESGO.125}

Introduction/Background* The purpose of this pilot study was to determine whether the MRI and biomarkers human epididymis protein 4 (HE4) and CA125 correlate with depth of myometrial invasion, histologic grade, cervical involvement and nodal metastases in patients with endometrioid adenocarcinoma of the uterus.

Methodology This was a prospective, observational study in women with biopsy-proven endometrial adenocarcinoma of the uterus. Preoperative pelvic MRI was performed and concentration of HE4 and CA125 were assessed before surgery. All surgical specimens were reviewed by a single expert

Abstract 198 Table 1 Relation between serum CA125 level, serum (HE4) level and histopathological prognostic factors

\begin{tabular}{|c|c|c|c|c|c|c|}
\hline & $\begin{array}{l}\text { HE4 < } \\
\text { 140PM [n } \\
(\%)]\end{array}$ & $\begin{array}{l}\text { HE4 } \geq \\
140 P M[n \\
(\%)]\end{array}$ & $p$ & $\begin{array}{l}\text { CA125< } \\
35 \mathrm{ku} / \mathrm{I}[\mathrm{n} \\
(\%)]\end{array}$ & $\begin{array}{l}\text { CA125 } \geq \\
35 \mathrm{ku} / \mathrm{I}[\mathrm{n} \\
(\%)]\end{array}$ & $\mathrm{p}$ \\
\hline FIGO & & & 0.003 & & & 0.002 \\
\hline 1 & 47 (90.4\%) & $5(9.6 \%)$ & & $38(73.1 \%)$ & $14(26.9 \%)$ & \\
\hline II & $6(66.7 \%)$ & $3(33.3 \%)$ & & $3(33.3 \%)$ & $6(66.7 \%)$ & \\
\hline III & $3(42.9 \%)$ & $4(57.1 \%)$ & & $1(14.3 \%)$ & $6(85.7 \%)$ & \\
\hline $\begin{array}{l}\text { Myometrial } \\
\text { invasion }\end{array}$ & & & 0.001 & & & 0.0001 \\
\hline No & $1(100 \%)$ & $0(0)$ & & $1(100 \%)$ & $0(0)$ & \\
\hline$<50 \%$ & $37(97.4 \%)$ & $1(2.6 \%)$ & & 32 (84.3\%) & $6(15.7 \%)$ & \\
\hline$\geq 50 \%$ & $18(62.1 \%)$ & 11 (37.9\%) & & $9(14.3 \%)$ & $6(68.9 \%)$ & \\
\hline Grade & & & 0.035 & & & 0.001 \\
\hline 1 & $27(96.5 \%)$ & $1(3.5 \%)$ & & $23(82.2 \%)$ & $5(17.8 \%)$ & \\
\hline II & 25 (71.5\%) & $10(28.5 \%)$ & & $19(31.1 \%)$ & 16 (45.7\%) & \\
\hline III & $4(80 \%)$ & $1(20 \%)$ & & $0(0)$ & $5(100 \%)$ & \\
\hline $\begin{array}{l}\text { Node } \\
\text { involvement }\end{array}$ & & & 0.007 & & & 0.002 \\
\hline No & $54(85.2 \%)$ & $8(14.8 \%)$ & & $42(66.7 \%)$ & $20(33.3 \%)$ & \\
\hline Yes & $2(33.4 \%)$ & $4(66.6 \%)$ & & $0(0)$ & $6(100 \%)$ & \\
\hline $\begin{array}{l}\text { Cervical } \\
\text { stromal } \\
\text { Involvement }\end{array}$ & & & & & & 0.006 \\
\hline No & 48 (88.9\%) & $6(11.1 \%)$ & & $38(70.4 \%)$ & $16(29.6 \%)$ & \\
\hline Yes & $8(57.2 \%)$ & $6(42.8 \%)$ & & $4(28.6 \%)$ & $10(71.4 \%)$ & \\
\hline
\end{tabular}

